\title{
Bacterial isolates from cerebrospinal fluids and their antibiotic susceptibility patterns in Gondar University Teaching Hospital, Northwest Ethiopia
}

\author{
Andargachew Mulu ${ }^{1}$, Afework Kassu ${ }^{1}$, Belay Tessema ${ }^{2}$
}

\begin{abstract}
Background: Bacterial meningitis remains a common disease worldwide. Its most frequent causes are Nessieria meningitidis, Streptococcus pneumoniae, and Haemophilus influenzae. Information on the relative frequency of the isolation and antibiotic susceptibility patterns of these pathogens is scarce in Ethiopia.

Objectives: To identify bacterial pathogens that cause meningitis and to assess the antibiotic susceptibility patterns of the isolates from the cerebrospinal fluids (CSF) of acute bacterial meningitis cases/specimens.

Methods: A retrospective analysis of 390 cerebrospinal fluid specimens submitted for culture and antibiotic susceptibility patterns to the bacteriology laboratory of Gondar University Teaching Hospital was conducted between September 2002 and August 2003.

Results: Bacterial pathogens were isolated from 22 patients showing an isolation rate of 5.6\%. The most commonly isolated bacteria were Neisseria meningitidis 10(45.5\%) and Streptococcus pneumoniae 7(31.8\%). Among gram positive organisms $S$. pneumoniae showed a high level of drug resistance against chloramphenicol 4(57\%), tetracycline $3(43 \%)$, co-trimoxazole 3(43\%), ampicillin 3(43\%), and gentamicin 1(14\%). Among gram negative bacteria, $N$. meningitidis was found to be resistant to co-trimoxazole 5(50\%), chloramphenicol 3(30\%), gentamicin 3(30\%) and ampicillin 2(20\%). The single isolate from Proteus species was found to be resistant to co-trimoxazole and tetracycline. E. coli was found to be resistant to all antibiotics except for gentamicin and ciprofloxacin. Multiple drug resistance was observed in $50 \%$ of the isolates. No organism was found to be resistant to ciprofloxacin.

Conclusions: The isolation rate of bacterial pathogens from cerebrospinal fluids was found to be low. However, the frequency of single as well as multiple drug resistance was very high among the bacterial isolates. Ciprofloxacin may be used for the empirical treatment of bacterial meningitis when culture and sensitivity report is not available for adult patients. [Ethiop.J.Health Dev. 2005; 19(2):160-164]
\end{abstract}

\section{Introduction}

Meningitis is a very serious infection of the meninges that surround the brain and the spinal cord (1). It is usually caused by viral, bacterial or fungal pathogens. Bacterial meningitis can be quite severe and may result in brain damage, hearing loss, or learning disability and death if not treated early (2). Despite advances in vaccine development and chemoprophylaxis, bacterial meningitis remains a common disease worldwide. The disease is more common in developing countries (3).

The relative frequency of isolation of various bacterial species as a cause of meningitis varies with age, and among geographical regions. About $80 \%$ of all cases of bacterial meningitis are caused by $N$. meningitidis, $S$. pneumoniae, and $H$. influenzae (3-5). Over two-thirds of all cases of bacterial meningitis occur in children less than five years old. Meningococcus affects all ages, most cases occurring in children and adolescents. More than $80 \%$ of meningitis caused by $H$. influenzae occurs in children less than 5 years old. Group B hemolytic streptococcus ( $S$. agalactiae) is the commonest cause of meningitis in neonates. E. coli is a frequent cause of meningitis in neonates and is rarely a cause after infancy (5).
The highest burden of meningococcal meningitis occurs in sub-Saharan Africa, in an area that is referred to as the meningitis belt, which has an estimated total population of approximately 300 million (3). Studies on the epidemiology of meningococcal meningitis in adult Ethiopians shows fatality rates for meningitis and meningococcemia to be $16 \%$ and $85 \%$ respectively (6).

Antimicrobial resistance of bacteria is a worldwide problem. However, the situation in developing countries like Ethiopia is particularly serious (7-8). Because of the absence of well-equipped bacteriological laboratories in Ethiopia no organized surveillance exists on drug resistance patterns among common bacterial isolates and thus published data on drug resistance are few. However, the presence multiple antimicrobial resistances in different clinical specimens including CSF have been indicated (9-15).

Since the presence of drug resistant bacteria in the natural environment is a great-threat for public health, and because of the ever increasing number of drug resistant strains with time, up-to-date information on local pathogens and their drug susceptibility patterns is very

\footnotetext{
${ }^{1}$ Department of Microbiology and Parasitology, Gondar University, P.O. Box 196, Tel. 251-08-11-01-74, EXT 307 Fax 251-08-111479, E-mail andargachewmulu@yahoo.com, Gondar, Ethiopia; ${ }^{2}$ Departments of Medical Laboratory Technology, Gondar University, P. O. Box 196, Gondar, Ethiopia
} 
crucial for the treatment of patients, to decide health priorities, to allocate resources, to monitor the onset of resistance and for planning the effective use of antimicrobials in the region. The purpose of this study was, therefore, to identify the prevalent bacterial pathogens that cause meningitis and to assess their antibiotic susceptibility patterns in Gondar University Teaching Hospital, northwest Ethiopia.

\section{Methods}

This Laboratory-based retrospective analysis of 390 CSF cultures and sensitivity tests was conducted in the Bacteriology Laboratory of the Department of Microbiology and Parasitology at Gondar University Teaching Hospital within a one-year period between September 2002 and August 2003.

CSF samples were collected as part of the routine clinical management of patients admitted in different wards of the hospital. The samples were collected in sterile containers by attending physicians and delivered to the Bacteriology laboratory within half an hour after collection.

The samples were processed following the standard microbiological procedures by inoculating on blood agar, chocolate agar, and MacConkey agar plates [(Oxoid Ltd, Basingstoke, Hampshire, UK) prepared as per the manufacturer instruction] and incubated at $35-37^{\circ} \mathrm{C}$ aerobically. The chocolate agar plates were incubated by putting them in a candle jar, which provided $5-10 \% \mathrm{CO}_{2}$ concentration to create a microareophilic condition for fastidious bacteria. After 18-20 hours of incubation, the plates were examined for the presence of bacterial colonies. Plates, which did not show any growth, were further incubated for an additional 24 hours. Organisms were identified by standard microbiological methods, which included colony morphology, as well as staining, biochemical and serological tests $(1,16-17)$.

Antibiotic sensitivity test was conducted on pure culture isolates employing the disc diffusion method (18) for the commonly used antibiotics: ampicillin $(10 \mu \mathrm{g})$, gentamicn $(10 \mu \mathrm{g})$, penicillin $\mathrm{G}(10 \mathrm{IU})$, tetracycline $(30 \mu \mathrm{g})$, chloramphenicol $(30 \mu \mathrm{g})$, ciprofloxacin $(5 \mu \mathrm{g})$ and cotrimoxazole $(25 \mu \mathrm{g})$ (Oxoid Ltd). The diameters of growth inhibition around the discs were measured and interpreted as sensitive, intermediate or resistant as per the guideline set by Bauer, et al (18). Reference strains: E. coli ATCC 25922 and S. aureus ATCC 25923 were tested as controls according to the National Committee for Clinical Laboratory Standards (NCCLS) (19).

\section{Results}

The sex and age distribution patterns of the study subjects are summarized in Figure 1. Out of the 390 suspected meningitis cases, 249 (63.8\%) were males and the remaining $141(36.2 \%)$ were females. The age range was from 3 days to 60 years. Over half of the 390 patients, $218(55.9 \%)$ were children and the rest $129(35.1 \%)$ and $35(9 \%)$ were adults and neonates, respectively.

Bacterial pathogens were isolated from 22 patients showing an isolation rate of $5.6 \%$. The number of pathogens isolated was equal in males and females. Pathogen isolation rate was higher in adults $8(5.8 \%)$ than in children $12(5.5 \%)$.

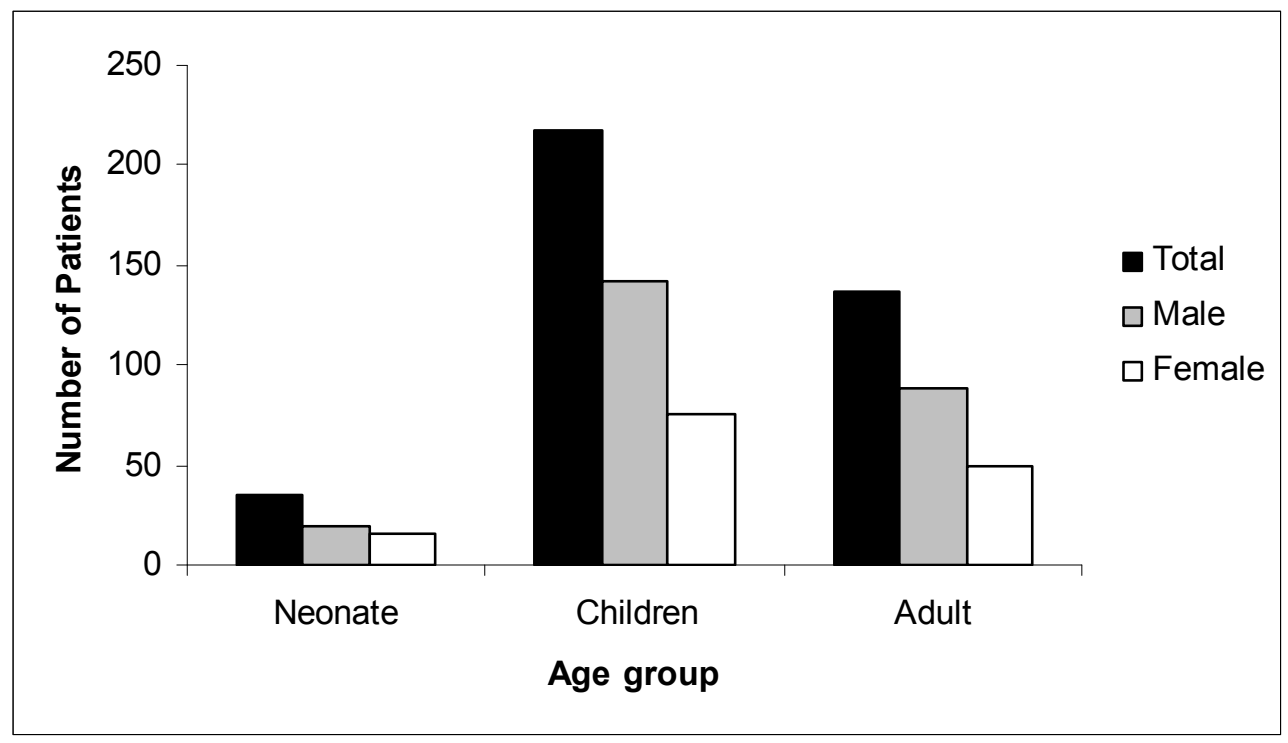

Figure 1: Age and sex distribution of patients investigated for meningitis in Gondar University Teaching Hospital, Sept. 2002-Aug. 2003 
The type and frequency of pathogens isolated from CSF is shown in Table 1. About 13 (59.1\%) of all the isolates were Gram negative organisms while $9(40.9 \%)$ were Gram positives. N. meningitidis was found to be the most frequent isolate $10(45.5 \%)$ followed by $S$. pneumoniae 7(31.8), S. aureus 2(9.1); H. influenzae, Proteus species and E. coli 1 (4.5\%) each. The majority of $N$. meningitidis $(70 \%)$ were isolated from children. The commonest isolate in adults was $S$. pneumoniae $3(43 \%)$. Multiple infections were not observed. All $S$. aureus, Proteus species and E. coli were isolated from adults (Table 1).

Table 1: Common bacterial isolates from CSF by sex and age distribution, Gondar University Teaching Hospital, September 2002-august 2003.

\begin{tabular}{llllllc}
\hline \multirow{2}{*}{ Organism } & \multirow{2}{*}{ Number (\%) } & Male & Female & Neonates & Children & Adults \\
\cline { 3 - 7 } & & 5 & 5 & 2 & 7 & 1 \\
N. meningitidis & $10(45.5)$ & 3 & 4 & 0 & 4 & 3 \\
S. pneumoniae & $7(31.8)$ & 1 & 1 & 0 & 0 & 2 \\
H. influenzae & $2(9.1)$ & $1(4.5)$ & 1 & 0 & 0 & 0 \\
Proteus species & $1(4.5)$ & 1 & 0 & 0 & 0 & 1 \\
E. coli & $1(4.5)$ & 1 & $\mathbf{1 1}$ & $\mathbf{2}$ & $\mathbf{1 2}$ & 1 \\
\hline Total & $\mathbf{2 2 ( 1 0 0 )}$ & $\mathbf{1 1}$ & & & $\mathbf{8}$
\end{tabular}

The results of the antibiotic susceptibility patterns are presented in Table 2 below. Among Gram positive organisms, S. pneumoniae showed a high level of drug resistance against chloramphenicol $4(57 \%)$, tetracycline $3(43 \%)$, co-trimoxazole $3(43 \%)$, ampicillin $3(43 \%)$, and gentamicin $1(14 \%)$. One $S$. aureus strain was found to be resistant only to ampicillin and the other showed an intermediate resistance to ampicillin, penicillin and tetracycline. Of the Gram-negative bacteria, $N$. meningitidis was found to be resistant to co-trimoxazole $5(50 \%)$, chloramphenicol $3(30 \%)$, gentamicin $3(30 \%)$ and ampicillin $2(20 \%)$. The single isolate of Proteus species was found to be resistant to co-trimoxazole and tetracycline. E. coli was found to be resistant to all antibiotics except for gentamicin and ciprofloxacin.

No organism was found to be resistant to ciprofloxacin. Seven $(31.8 \%)$ of the isolated pathogens were found to be sensitive to all antibiotics tested. Four (18\%), $5(22.7 \%)$, and $2(9 \%)$ of the isolated pathogens were found to be resistant to one, two and three antibiotics respectively. Resistance to four and five different antibiotics was found in $3(13.6 \%)$ and $1(11 \%)$ of the isolates, respectively.

Table 2: Antibiotic susceptibility pattern of bacterial isolates from CSF, Gondar University Teaching Hospital, September 2002 - August 2003.

\begin{tabular}{|c|c|c|c|c|c|c|c|c|}
\hline \multirow[t]{2}{*}{ Organisms } & & \multicolumn{7}{|c|}{ Antimicrobial agent } \\
\hline & & $\begin{array}{l}\text { Amp } \\
\text { No (\%) }\end{array}$ & $\begin{array}{l}\text { Gen } \\
\text { No (\%) }\end{array}$ & $\begin{array}{l}\text { Pen } \\
\text { No (\%) }\end{array}$ & $\begin{array}{l}\text { Sxt } \\
\text { No (\%) }\end{array}$ & $\begin{array}{l}\text { TTC } \\
\text { No (\%) }\end{array}$ & $\begin{array}{l}\text { CAF } \\
\text { No (\%) }\end{array}$ & $\begin{array}{l}\text { Cip } \\
\text { No (\%) }\end{array}$ \\
\hline \multirow[t]{3}{*}{ N. meningitidis 10 (45.5) } & $\mathbf{S}$ & $8(80)$ & $6(60)$ & - & $2(20)$ & $5(50)$ & $6(60)$ & $9(90)$ \\
\hline & I & - & $1(10)$ & - & $3(30)$ & $2(20)$ & $1(10)$ & $1(10)$ \\
\hline & $\mathbf{R}$ & $2(20)$ & $3(30)$ & - & $5(50)$ & $3(30)$ & $3(30)$ & - \\
\hline \multirow[t]{3}{*}{ S. pneumoniae 7 (31.8) } & $\mathbf{s}$ & $4(47)$ & $3(43)$ & $7(100)$ & $3(43)$ & $3(43)$ & $3(43)$ & $7(57)$ \\
\hline & I & - & $3(43)$ & - & 1 (14) & $1(14)$ & - & - \\
\hline & $\mathbf{R}$ & $3(43)$ & $1(14)$ & - & $3(43)$ & $3(43)$ & $4(57)$ & - \\
\hline \multirow[t]{3}{*}{ S. aureus 2 (9.1) } & $\mathbf{s}$ & - & $2(100)$ & $1(50)$ & $2(100)$ & $2(100)$ & $2(100)$ & $2(100)$ \\
\hline & I & $1(50$ & - & $1(50)$ & - & - & - & - \\
\hline & $\mathbf{R}$ & $1(50$ & - & - & - & - & - & - \\
\hline \multirow[t]{3}{*}{ H. Influenzae 1 (4.5) } & $\mathbf{s}$ & $1(100)$ & $1(100)$ & - & $1(100)$ & $1(100)$ & $1(100)$ & $1(100)$ \\
\hline & I & - & - & $1(100)$ & - & - & - & - \\
\hline & $\mathbf{R}$ & - & - & - & - & - & - & - \\
\hline \multirow[t]{2}{*}{ Proteus species 1 (4.5) } & $\mathbf{s}$ & $1(100)$ & $1(100)$ & - & - & $1(100)$ & $1(100)$ & $1(100)$ \\
\hline & $\mathbf{R}$ & - & - & - & $1(100)$ & - & - & $-\quad$ \\
\hline \multirow[t]{2}{*}{ E. coli $1(4.5)$} & $\mathbf{s}$ & - & $1(100)$ & - & & - & - & $1(100)$ \\
\hline & $\mathbf{R}$ & $1(100)$ & - & - & $1(100)$ & $1(100)$ & $1(100)$ & - \\
\hline
\end{tabular}

Key; Amp=Ampicillin, Pen=Penicillin, Gen=Gentamycin, Sxt=Co-trimoxazole, TTC=Tetracycline, $\mathrm{CAF}=$ Chloramphenicol, $\mathrm{Cip}=$ Ciprofloxacin $\mathrm{S}=$ sensitive, I=Intermediate, $\mathrm{R}=$ Resistant 


\section{Discussion}

The successful management of patients suffering from bacterial illnesses depends upon the identification of the types of organisms that cause the diseases and the selection of an effective antibiotic against the organism in question (16). Thus, the data presented in this study could provide information of immediate public health importance to clinicians in northwest Ethiopia on the selection of antimicrobial agents for the treatment of patients suffering from acute bacterial meningitis.

The finding of this retrospective study indicated that the spectrum of bacterial isolates from CSF in general is basically similar to reports from elsewhere (2-3). N . meningitidis and $S$. pneumoniae were the commonest pathogens of meningitis patients. The isolation rate of $5.6 \%$ found in the present study is in agreement with a previous study conducted in Gondar where an isolation rate of 5.2\% was reported (9). Eventhough all febrile children and adults without localizing signs have underwent lumbar puncture to rule out bacterial meningitis, the bacterial isolation rate was found to be low. This may be due to the fastidious nature of the organisms or prior exposure to antibiotics $(1,5,8)$ or over clinical diagnosis of meningitis.

Eighty one percent of cases of acute bacterial meningitis were caused by $N$. meningitidis, $S$. pneumoniae and $H$. influenzae. Seventy percent of meningococci were isolated from children, which is in agreement with CDC and WHO reports (2-3). However, this is in contrast with a previous study conducted in Gondar where no meningococci were isolated (9). The isolation rate of the menigococci in the present report might be a result of an epidemic of meningococcal meningitis which occurred in North Gondar in 2001/ 2002 (21).

H. influenzae have been reported as the most common pathogen of pyogenic meningitis in children in central and northwestern Ethiopia $(15,20,24)$. The reason for the existence of only a single isolation of $H$. influenzae over the year in the present study is not clear. However, it might be due to the new vaccine being given as part of routine child immunization activities, which might have reduced the occurrence of invasive diseases due to $H$. influenzae (3) and/or the more fastidious nature of the organism $(1,5,16)$. Unlike previous studies conducted in the same area (21), the common causative organism for neonatal meningitis in the present study was found to be $N$. meningitidis. However, it is in agreement with a previous study conducted in Addis Ababa (23-24).

Resistance of $S$. pneumoniae, $N$. meningitidis and $H$. influenzae to commonly prescribed antibiotics has been reported in different areas of the country (15). But much is not known about the drug resistance behaviour of $S$. pneumoniae and $N$. meningitidis from northwest Ethiopia. Nevertheless, Achamyeleh et al. (14) in 1995 reported that $S$. pneumoniae isolated from pediatric patients were found to be resistant to the commonly prescribed antibiotics. In agreement to this report S. pneumoniae in the present study were found to have high levels of drug resistance against chloroamphenicol, tetracycline, cotrimoxazole, ampicillin, and gentamicin. However, no $S$. pneumoniae were found to be resistant to penicillin unlike a previous report from the same area (15) where 50 percent penicillin resistant pneumococcal meningitis type was reported. Likewise a high rate of resistance was also observed in N. meningitidis in the present study.

Previous S. aureus isolates from Gondar (12-13) showed similar frequencies of resistance to the common antibiotics tested. On the contrary, better susceptibility was observed among $S$. aureus strains to penicillin $G$ in the present study. The single isolate of $E$. coli was found to be sensitive only to gentamicin and ciprofloxacin. And Proteus species was found to be susceptible to ampicillin, gentamicin, chloramphenicol and ciprofloxacin. These are in agreement with previous reports in studies from Jimma and Gondar (9-10, 12-13). In general, multiple drug resistance was seen in $50 \%$ of the bacterial isolates from CSF. No organism was found to be resistant to ciprofloxacin as reported earlier (12-13).

In conclusion the isolation rate of bacteria causing meningitis is low. This may be probably due to the fastidious nature of the organisms, prior exposure to antibiotics or over clinical diagnosis of meningitis, especially in children. The frequency of single as well as multiple drug resistance is alarmingly high. This might be a reflection of the inappropriate use of antimicrobials, or unavailability of a guideline regarding the selection of drugs. Producing updated information on local pathogens and their sensitivity patterns is a prime tool in combating such a global problem. Since no resistance was seen against ciprofloxacin, this antibiotic may be used for the immediate empirical therapy of meningitis before culture and sensitivity report is available, particularly for adult patients.

\section{Acknowledgements}

The authors are grateful to the Medical Laboratory Technicians working in the Bacteriology section of the laboratory and to the Department of Clinical Laboratory for giving access to their documentation and to the staff of the Microbiology Department of the College for their valuable comments.

\section{References}

1. Cheesborough M. Medical Laboratory Manual for Tropical countries II. 1987: 255 - 275.

2. Richard E.B., et al. Nelson textbook of pediatrics, $16^{\text {th }}$ ed. W.B. Saunders Company. New York USA, 2000. P.707. 
3. World Health Organization. Control of epidemic meningococcal diseases. WHO Practical Guidelines. 2 ed. 1998. Geneva Switzerland.

4. Solberg C.O. Meningococcal infections, In Fauci A.S., Braunwald E, Isselbacher K.J, et al (eds.), Harrison's principles of internal medicine, $14^{\text {th }}$ ed. McGraw- Hill, New York USA 1998; 9:910-15.

5. D.C. Shanson. Microbiology in Clinical Practice $3^{\text {rd }}$ ed. Butterworth Heinemann: 305-306.

6. Fekade D., Zewde D., Epidemic meningococcal meningitis in adult Ethiopians in Addis Ababa, Ethiopia. Ethiop Med J 1992;30(3):135-42.

7. World Health Organization. WHO Bulletin. Resistance to antimicrobial agents 1996;74(3):335336.

8. Robert F. Basic Medical Microbiology. 5th ed. Lippincott Williams and Wilkins Country of Publication. 252-267.

9. Aseffa A, Yohannes G. Antibiotic sensitivity patterns of prevalent bacterial pathogens in Gondar, Ethiopia. East Afr Med J. 1996;73(1):67-71.

10. Beyene G, Abdissu T. Common bacterial pathogens and their antibiotic sensitivity at Jimma Hospital: A four-year Retrospective study. Ethiop J Health Sci 2000;10(2):129-35.

11. Roma B, Worku S, T/Mariam S, Langeland N. Antimicrobial susceptibility pattern of Shigella isolates in Awassa. Ethiop J. Health Dev 2000;14(2): 149-154.

12. Moges F, Genetu A, Mengistu G. Antibiotic sensitivity of common bacterial pathogens in urinary tract infections at Gondar Hospital, Ethiopia. East Afr Med J 2002;79(3):140-142.

13. Moges F, Mengistu G, Genetu A. Multiple drug resistance in urinary pathogens at Gondar College of Medical Science Hospital, Ethiopia. East Afr Med J 2002;79(8):415-419.

14. Achamyeleh A, Aseffa A, Gedlu E. Bacterial isolates from CSF over a five year period in Gondar with a special reference to pediatric admissions and out come. Proceeding of an Annual Research conference, Gondar College of Medical Sciences. 1995:51-58.
15. Muhe L, Klugman KP. Pneumococcal and Haemophilus infleunzae meningitis in a children's Hospital in Ethiopia: Serotypes and susceptibility pattern. Trop Med Int Health 1999;4(6):421-7.

16. Jawetz, Melnick, Adelbergis. Medical Microbiology. 19 Ed. Prentice-Hall International Inc: Antibacterial and Antifungal chemotherapy.149-179.

17. World Health Organization. Basic laboratory procedures in clinical bacteriology, WHO, Geneva, Switzerland, 1991:78-95.

18. Bauer, A.W., Kirby, W.M.M., Sherirs, J.C, Turck, M. Antibiotic susceptibility testing by standard single disk method. Am J Clin Pathol 1966;45:433496.

19. National Committee for Clinical Laboratory Standards. Performance standards for antimicrobial disc susceptibility tests. Approved Standard, ASM-2 ( $2^{\text {nd }}$ ed.) NCCLS, Villanovan, pa. 1979.

20. Gedlu E, Rahlenbeck SI. Pyogenic meningitis in children in northwest Ethiopia. Ann Trop Paediatr 1995;15(3):243-247.

21. Mengistu G, Mitiku K, Teferi W. Analysis and reporting of meningococcal meningitis epidemic in north Gondar. Ethiop Med J 2003;41(4):319-31.

22. Gebremariam A. Neonatal meningitis in Addis Ababa: A 10-year review. Ann Trop Paediatr. 1995;15(3):243-247.

23. Kristos TG, Muhe L. Epidemic meningococcal meningitis in children. A retrospective analysis of cases admitted to ESCH. Ethiop Med J 1993;31(1): 9-14.

24. Hailu M, Muhe L. Childhood meningitis in a tertiary hospital in Addis Ababa: Clinical and epidemiological features. Ethiop Med J 2001;39(1): 29-38. 